\title{
A New Image Sharpening Approach for Single-Sensor Digital Cameras
}

\author{
Rastislav Lukac, ${ }^{1}$ Konstantinos N. Plataniotis ${ }^{2}$ \\ ${ }^{1}$ Epson Edge, Epson Canada Ltd., M1W $3 Z 5$ Toronto, Ontario, Canada \\ ${ }^{2}$ The Edward S. Rogers Sr. Department of ECE, University of Toronto, Toronto, Ontario, M5S \\ 3G4, Canada
}

Received 25 February 2007; accepted 30 August 2007

\begin{abstract}
This article introduces a new image sharpening approach suitable for single-sensor digital cameras equipped with a Bayer color filter array (CFA). The proposed solution firstly enhances the structural content of the captured CFA image data. Subsequent demosaicking of the enhanced CFA image data produces a visually pleasing full-color image which is noticeably sharper compared to the output of the traditional imaging pipeline. Results reported in this work suggest a three-fold processing cost reduction when the new approach is followed. ( 2007 Wiley Periodicals, Inc. Int J Imaging Syst Technol, 17, 123-131, 2007; Published online in Wiley InterScience (www.interscience.wiley.com). DOI 10.1002/ima.20111
\end{abstract}

Key words: digital color imaging; single-sensor camera; color filter array; Bayer pattern; demosaicking; image sharpening

\section{INTRODUCTION}

Image-enabled consumer electronic devices, such as digital cameras, mobile phones, and wireless personal digital assistants (PDAs) use a color filter array (CFA) placed on top of a single image sensor to capture the visual scene (Lukac and Plataniotis, 2005). Since the sensor, usually a charge-coupled device (CCD) or complementary metal oxide semiconductor (CMOS) sensor, is a monochrome device and each sensor cell has its own spectrally selective filter, the acquired CFA data constitutes a mosaic-like, gray-scale image. The true color information is restored by a demosaicking process which uses spectral interpolation to estimate the missing color components at each spatial location of the CFA image, thus producing a fullcolor demosaicked image (Longére et al., 2002; Gunturk et al., 2005; Lukac and Plataniotis, 2005).

Various visual artifacts such as aliasing and color shifts are often present in the demosaicked output (Fig. 1a) due to the under-sampling that CFA performs to the original color information of the captured visual scene. To reduce these effects (Fig. 1b), camera manufacturers place a blurring filter in the optical path (Parulski and Spaulding, 2002). However, the filter reduces both the sharp-

Correspondence to: Rastislav Lukac; e-mail: lukacr@ieee.org

Web: www.colorimageprocessing.com ness and the resolution of the captured image. Additional blur is often introduced to the captured image by the sensor and due to the camera motion and insufficient focusing. To compensate for the image blurring, digital cameras enhance the visual quality of the demosaicked output using image sharpening techniques (Parulski and Spaulding, 2002; Ramanath et al., 2005). Image sharpening utilizes high-pass type operations to enhance the high-frequency content of the image, such as edges and fine details (Polesel, et al., 2000; Fischer et al., 2002). Since edge information is very important for the human perception (Bouzit and MacDonald, 2000), its preservation and possibly enhancement is of paramount importance for any imaging system (Koschan and Abidi, 2005; Lukac et al., 2005).

In this article, we propose a framework which enhances the structural content of the acquired CFA image data by employing an image sharpening step before the actual demosaicking module. This constitutes a cost-effective processing solution capable of producing sharp demosaicked images without the need for expensive sharpening in the red-green-blue (RGB) color domain. Extensive experimentation reported in this work indicates that the proposed framework outperforms traditional approaches used to sharpen demosaicked images in terms of both performance and computational efficiency.

This article is organized as follows. Section II presents both the conventional and the proposed camera image sharpening approaches. Motivation and design characteristics are introduced and discussed in detail. In Section III, the proposed CFA image sharpening framework is tested using a variety of standardized test images as well as real-life raw camera CFA images. The framework is also compared against the traditional demosaicked image sharpening solution. The computational complexity of the proposed approach is analyzed in Section IV. Finally, this article is concluded in Section V.

\section{CAMERA IMAGE SHARPENING}

The data acquired by the sensor with the CFA constitutes a $K_{1} \times K_{2}$ gray-scale mosaic-like image $z$ with the pixels $z_{(r, s)}$. Occupying the 

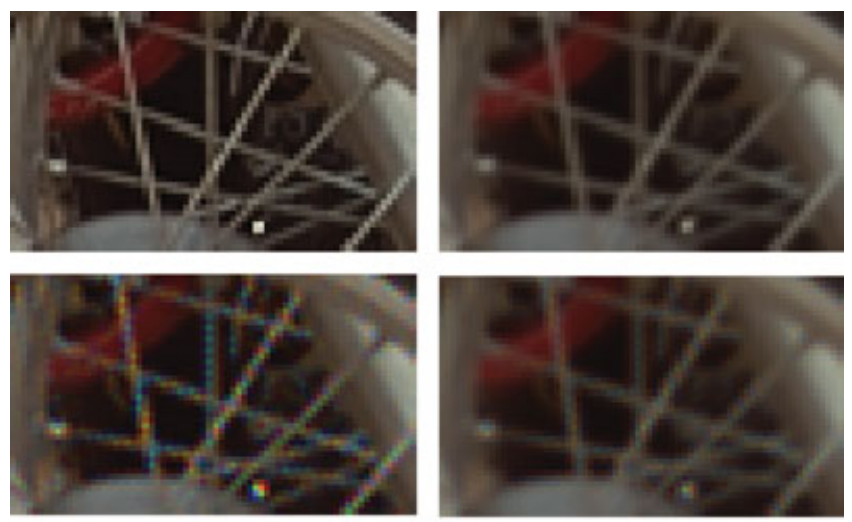

(a)

(b)

Figure 1. Simulation of the visual scene (top) and the corresponding demosaicked images (bottom) obtained using bilinear interpolation for a single-sensor camera operating: (a) without, and (b) with the blurring/antialiasing filter in the optical path. [Color figure can be viewed in the online issue, which is available at www.interscience. wiley.com.]

spatial location $(r, s)$, with the row coordinate $r=1,2, \ldots, K_{1}$ and the column coordinate $s=1,2, \ldots, K_{2}$, the integer value of $z_{(r, s)}$ is ranged from 0 to 255 in standard 8-bits per pixel representation. Using a popular Bayer CFA with the GRGR phase in the first row (Fig. 2) (Bayer, 1976), $z_{(r, s)}$ corresponds to the R component for (odd $r$, even $s$ ), the G component for (odd $r$, odd $s$ ) and (even $r$, even $s$ ), and the B component for (even $r$, odd $s$ ).

A. Demosaicked Image Sharpening. The CFA image $z$ is demosaicked (Gunturk et al., 2005; Lukac and Plataniotis, 2005) to produce a $K_{1} \times K_{2}$ RGB color image $\mathbf{x}$ which constitutes a two-dimensional matrix of three-component samples $\mathbf{x}_{(r, s)}=$ $\left[x_{(r, s) 1}, x_{(r, s) 2}, x_{(r, s) 3}\right]^{T}$. The pixel $\mathbf{x}_{(r, s)}$ represents the color vector where the value of its $\mathrm{R}(k=1), \mathrm{G}(k=2)$, and $\mathrm{B}(k=3)$ component $x_{(r, s) k}$ denotes the contribution of the $k$ th primary in $\mathbf{x}_{(r, s)}$.

After demosaicking the acquired CFA data, the demosaicked image is often postprocessed in order to reduce various visual artifacts introduced during demosaicking (Lukac et al., 2004b). The visual appearance of the captured image is usually enhanced using color correction, white balance, tonescale rendering, sharpening, and noise filtering. An overview of these processing steps can be found in (Parulski and Spaulding, 2002; Ramanath et al., 2005).

The perceived sharpness of the captured image is improved by enhancing color and tonal changes and transitions. In off-camera sharpening, such as for web and monitor viewing and print reproduction, the edge enhancement process is often manually controlled by tuning the parameters in various unsharp masking and orderstatistic solutions (Polesel, et al., 2000; Fischer et al., 2002) to avoid both the introduction of sharpening halos and the increase in the visibility of jaggedness, noise, and various demosaicking artifacts. However, in-camera sharpening requires different solutions due to the real-time constraints and the need for fully automated processing. Therefore, digital camera manufacturers use simple and easy to implement solutions such as the Laplacian high-pass filters (Parulski and Spaulding, 2002) which enhance the color image $x$ as follows: $x_{(r, s) k}^{\prime}=x_{(r, s) k}+\sum_{(i, j) \in \zeta}\left\{w_{(i, j)} x_{(i, j) k}\right\}$.

Figure 3 a shows a common $3 \times 3$ window with the area of support $\zeta$ and the coefficients $w_{(i, j)}$ of the high-pass filter. The signal produced by $\sum_{(i, j) \in \zeta}\left\{w_{(i, j)} x_{(i, j) k}\right\}$ is a filtered high-frequency component of the local neighborhood $\zeta$ centered in the $(r, s)$ pixel location. This component is added to the original signal $x_{(r, s) k}$ to produce the enhanced signal $x_{(r, s) k}^{\prime}$. Sharpening the image $\mathbf{x}$ by the so-called demosaicked color image sharpener (DCIS) requires that the filtering procedure is repeated for $r=1,2, \ldots, K_{1}, s=1,2, \ldots, K_{2}$, and $k=1,2,3$, resulting in a $K_{1} \times K_{2}$ color image $\mathbf{x}^{\prime}$ with the pixels $\mathbf{x}_{(r, s)}^{\prime}=\left[x_{(r, s) 1}^{\prime}, x_{(r, s) 2}^{\prime}, x_{(r, s) 3}^{\prime}\right]^{T}$.

B. CFA Image Sharpening. Because of the use of CFA in the imaging pipeline, sharpening in the demosaicked color domain is not the only option to enhance the captured image (Alleysson et al., 2006; Lukac and Plataniotis, 2006). By operating directly on the CFA image using $z_{(r, s)}^{\prime}=z_{(r, s)}+\sum_{(i, j) \in \zeta}\left\{w_{(i, j)} z_{(i, j)}\right\}$ as proposed in this work, the enhanced data $z_{(r, s)}^{\prime}$ is comprised of the original CFA entry $z_{(r, s)}$ and the value of $\sum_{(i, j) \in \zeta}\left\{w_{(i, j)} z_{(i, j)}\right\}$ which is the output of the high-pass filter operating over CFA samples $z_{(i, j)}$ in the neighborhood $\zeta$. In this way, the sharpening process does not amplify the visual impairments introduced by demosaicking and generates visually pleasing, sharp, demosaicked images.

Since the Bayer CFA consists of three different color filters arranged in the mosaic layout, sharpening solutions should operate on the $z_{(i, j)}$ entries corresponding to the same color channel to avoid processing errors due to the amplification of the underlying mosaic. Thus, following the arrangement of color filters in the Bayer CFA (Fig. 2), the mask $\zeta$ from Figure 3a is inappropriate for CFA image sharpening because this mask enforces the calculation of the highpass filtered signal using CFA entries corresponding to different color channels. To overcome the problem, a $3 \times 3$ mask comprised of nine pixels in Figure 3 a should be extended to its $5 \times 5$ ninepoint variant shown in Figure $3 b$ which is used in the first proposed CFA sharpener $\left(\right.$ CFAS $\left._{1}\right)$.

Visual inspection of the Bayer CFA shown in Figure 2 suggests that the quincunx layout of $\mathrm{G}$ locations offers more than one possibility for the enhancement. For example, the proposed $\mathrm{CFAS}_{2}$ solution uses all G CFA locations in a $5 \times 5$ neighborhood, as shown in Figure 3c. On the other hand, the proposed $\mathrm{CFAS}_{3}$ solution uses $\mathrm{G}$ CFA locations in a $3 \times 3$ neighborhood depicted in Figure $3 \mathrm{~d}$. Note that for all masks listed in Figure 3 the sum of the elements is zero, that is, $\sum_{(i, j) \in \zeta} w_{(i, j)}=0$. This constraint is needed to avoid intensity bias in the enhanced image.

By applying the filter depicted in Figure $3 \mathrm{~b}$ for $r=1,2, \ldots, K_{1}$ and $s=1,2, \ldots, K_{2}$ in the proposed framework, the CFAS 1 solution

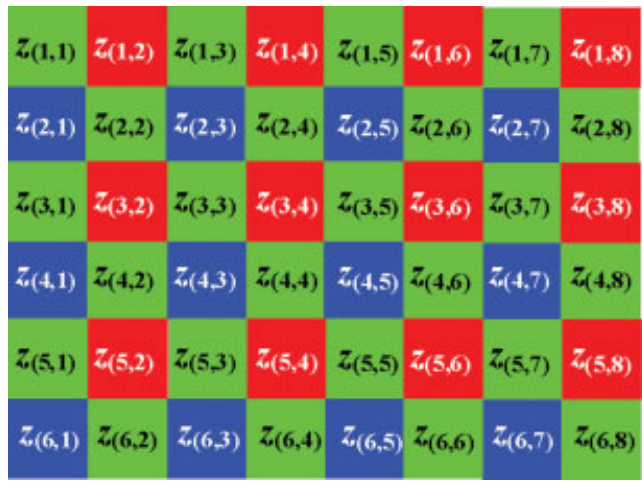

color

filters:

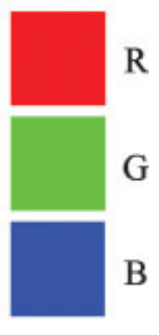

Figure 2. Bayer CFA with the GRGR phase in the first row. [Color figure can be viewed in the online issue, which is available at www.interscience.wiley.com.] 


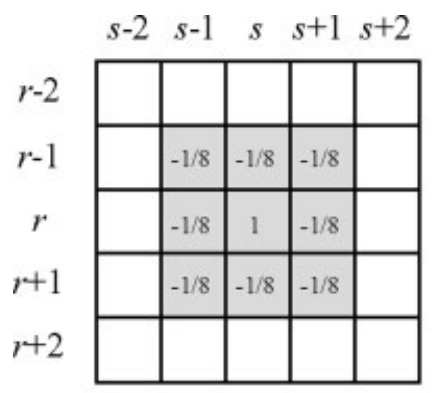

(a)

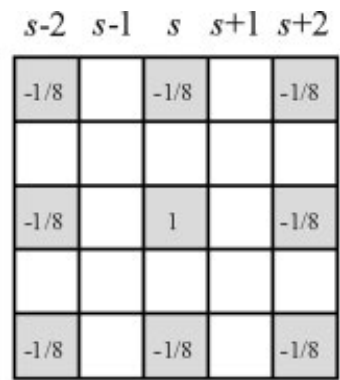

(b)

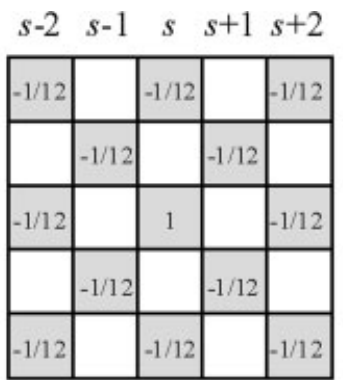

(c)

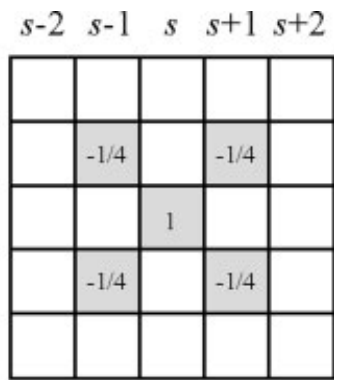

(d)

Figure 3. Laplacian high-pass filters with the weights $w_{(i, j)}$, for $(i, j) \in \zeta$, listed in the patterns. The filters use the following shape masks: (a) $\zeta=$ $\{(r-1, s-1),(r-1, s),(r-1, s+1),(r, s-1),(r, s),(r, s+1),(r+1, s-1),(r+1, s),(r+1, s+1)\},(b) \zeta=\{(r-2, s-2),(r-2, s),(r-2, s+2)$, $(r, s-2),(r, s),(r, s+2),(r+2, s-2),(r+2, s),(r+2, s+2)\}(c) \zeta=\{(r-2, s-2),(r-2, s),(r-2, s+2),(r-1, s-1),(r-1, s+1)$, $(r, s-2),(r, s),(r, s+2),(r+1, s-1),(r+1, s+1),(r+2, s-2),(r+2, s),(r+2, s+2)\},(d) \zeta=\{(r-1, s-1),(r-1, s+1),(r, s),(r+1, s-1)$, $(r+1, s+1)\}$.

generates a $K_{1} \times K_{2}$ enhanced CFA image $z^{\prime}$ with gray-scale values $z_{(r, s)}^{\prime}$. The same filter is also used by both $\mathrm{CFAS}_{2}$ and $\mathrm{CFAS}_{3}$ solutions in R CFA locations for $r=1,3, \ldots, K_{1}-1$ and $s=2,4, \ldots, K_{2}$, and in B CFA locations for $r=2,4, \ldots, K_{1}$ and $s=1,3, \ldots, K_{2}-1$. However, at all remaining locations (i.e., for $r=1,3, \ldots, K_{1}-1$, $s=1,3, \ldots, K_{2}-1$ and $\left.r=2,4, \ldots, K_{1}, s=2,4, \ldots, K_{2}\right), \mathrm{CFAS}_{2}$ and $\mathrm{CFAS}_{3}$ achieve sharpening by enhancing the G CFA values using the filters shown in Figure 3c and Figure 3d, respectively. After the CFA enhancement step is completed, the produced enhanced CFA image $z^{\prime}$ is processed by the demosaicking solution employed in the processing pipeline to generate the enhanced demosaicked image $\mathbf{x}^{\prime}$ with pixels $\mathbf{x}_{(r, s)}^{\prime}$. Since demosaicking is applied to the sharpened CFA image, no additional sharpening in the demosaicked color domain is necessary. The proposed processing pipeline directly outputs the enhanced color image with visually sharp edges and fine details.

C. Discussion. The DCIS and CFAS final outputs $\mathbf{x}_{(r, s)}^{\prime}$ are usually different from each other due to the reverse order of the processing steps and the different spatial masks $\zeta$ used to produce high-pass filtered signals. This suggests that the equivalence of both frameworks can appear only in smooth regions. In the edge areas, $\mathrm{CFAS}_{3}$ may not provide as such an amount of the enhancement as noticed for DCIS, CFAS ${ }_{1}$, and $\mathrm{CFAS}_{2}$, because $\mathrm{CFAS}_{3}$ has the smallest area of support among the solutions depicted in Figure 3. On the other hand, $\mathrm{CFAS}_{2}$ may not preserve well fine details because it operates using the large mosaic-like window.

Considering the filter characteristics; the DCIS enhanced signal should be much closer than the CFAS enhanced signal to an ideal high-pass filtered signal since the mask employed in DCIS (see Fig. 3a) is compact and does not have to follow the mosaic layout, as opposed to the masks used in CFAS (see Figs. 3b-3d). Detailed inspection of the amplitude spectra shown in Figure 4 reveals that DCIS is the only solution among the considered filters which exhibits high-pass characteristics in all directions (see Fig. 4a). Although CFAS high-pass characteristics (see Figs. 4b-4d) are not as compact as in DCIS, they still give the proposed framework sufficient edge enhancement ability.

Considering the implementation issues; because of the small area of support, $\mathrm{CFAS}_{3}$ is more computationally efficient than CFAS $_{1}$ and CFAS ${ }_{2}$ which is the most demanding solution within the proposed framework due to the largest number of the inputs used during enhancement. However, since each channel of the demosaicked image represents a monochrome image alike the acquired CFA image, the use of the CFA image sharpening framework basically results in a three-fold reduction of the processing cost compared to the equivalent solution applied to the demosaicked image. Therefore, it is expected that even $\mathrm{CFAS}_{2}$ is more efficient than DCIS.

Apart from the configurations shown in Figures 3b-3d, the proposed CFAS framework allows flexibly the use of various masks, with both fixed and adaptively determined coefficients, since the determination of the CFA inputs and the calculations of the enhanced output are solely based on the area of support $\zeta$ and the mask's coefficients $w_{(i, j)}$. Finally, it should be noted that this work aims to introduce a new camera image sharpening framework and compare its performance against the traditional solution. Therefore, only CFAS solutions which are dual to traditional DCIS solution are considered in this work to meet the objective and ensure fair comparisons. The enumeration of all available options or the

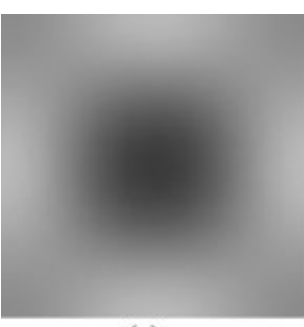

(a)

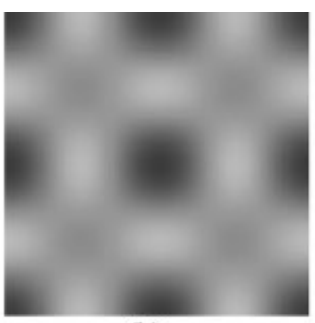

(b)

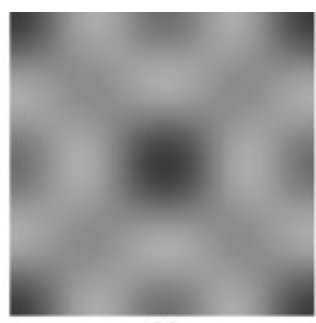

(c)

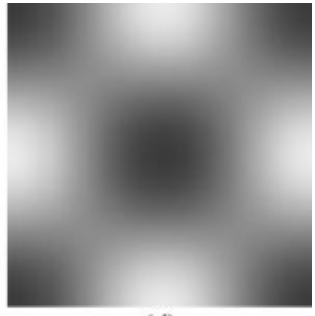

(d)
Figure 4. Amplitude spectra of the sharpeners under consideration: (a) DCIS, (b) $\mathrm{CFAS}_{1}$, (c) $\mathrm{CFAS}_{2}$, and (d) $\mathrm{CFAS}_{3}$. 


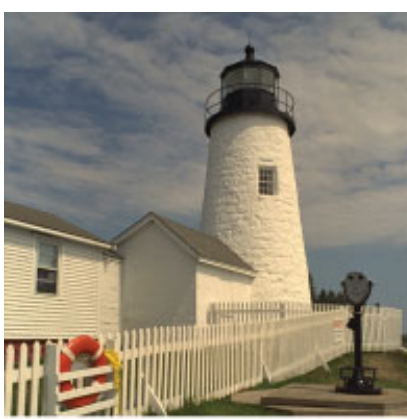

(a)

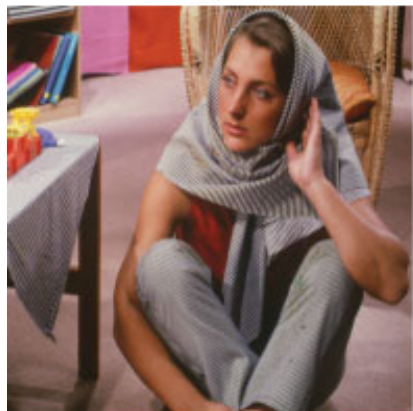

(e)

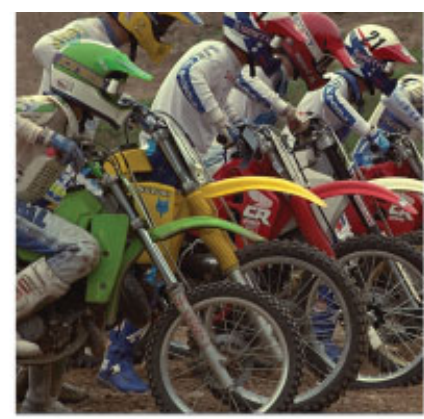

(b)

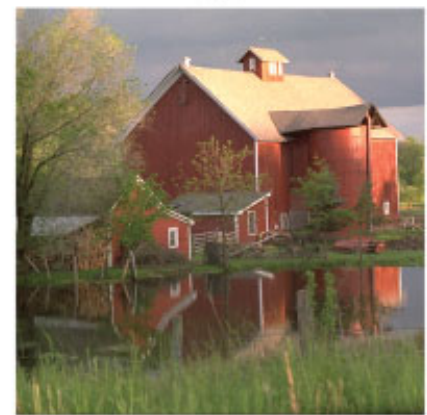

(f)

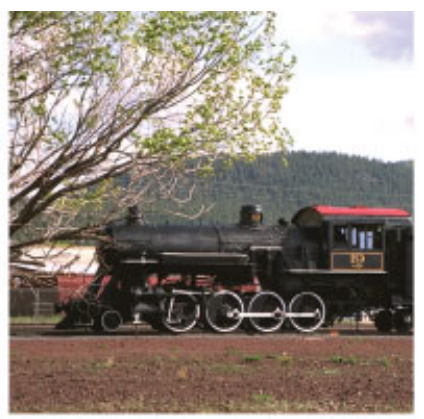

(c)

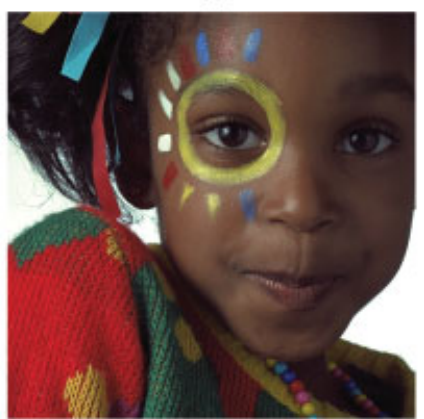

(g)

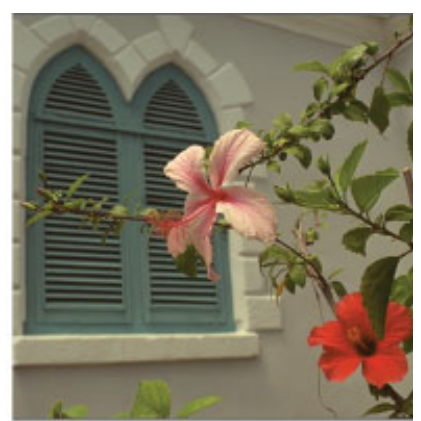

(d)

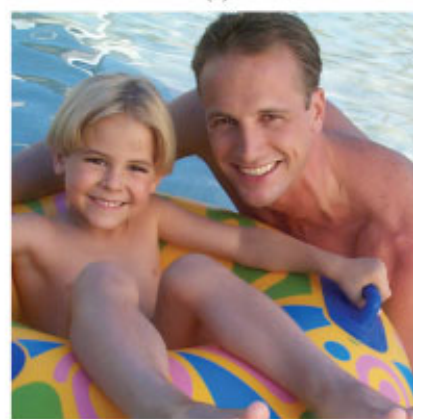

(h)

Figure 5. Test color images: (a) Lighthouse, (b) Bikes, (c) Train, (d) Window, (e) Barbara, (f) Solitude, (g) Girl, (h) Water. [Color figure can be viewed in the online issue, which is available at www.interscience.wiley.com.]

determination of the best configuration of construction elements, according to specified criteria, is beyond the scope of the paper.

\section{EXPERIMENTAL RESULTS}

To evaluate the performance of proposed camera image sharpeners, a number of test images have been utilized. Examples, shown in Figure 5, are used to emulate the application scenario. The test images which vary in color appearance and the complexity of edge information have been captured using a three-sensor device and normalized to an 8-bits per channel RGB representation with a $512 \times 512$ pixel resolution.

A. Experimentation Using Blurred Test Images. Since this work intends to simulate the real single-sensor imaging pipeline which uses the blurring/antialiasing filter prior to the acquisition of the data by the CFA sensor module, the test images are blurred before sampling the visual information using the CFA. Following the evaluation protocol depicted in Figure 6, tests were performed by blurring the $K_{1} \times K_{2}$ original images $\mathbf{o}^{\prime}$ with a $3 \times 3$ low-pass filter via $o_{(r, s) k}=\sum_{(i, j) \in \zeta}\left\{q_{(i, j)} o_{(i, j) k}^{\prime}\right\} / \sum_{(i, j) \in \zeta} q_{(i, j)}$, where $\zeta$ shown in Figure 3a denotes the local neighborhood defined over the original color vectors $\mathbf{o}_{(i, j)}^{\prime}=\left[o_{(i, j) 1}^{\prime}, o_{(i, j) 2}^{\prime}, o_{(i, j) 3}^{\prime}\right]^{T}$ and $q_{(i, j)}$ denotes the coefficients of the low-pass filter. Repeating the above procedure for $r=1,2, \ldots, K_{1}, s=1,2, \ldots, K_{2}$, and $k=1,2,3$ produces a $K_{1} \times K_{2}$ blurred color image $\mathbf{o}$ with the pixels $\mathbf{o}_{(r, s)}=\left[o_{(r, s) 1}, o_{(r, s) 2}, o_{(r, s) 3}\right]^{T}$. The blurred color image $\mathbf{o}$ was sampled with the GRGR-phased Bayer CFA (Fig. 2) to obtain a CFA image $z$. Thus, the CFA image data is given by $z_{(r, s)}=o_{(r, s) 1}$ for (odd $r$, even $s$ ), $z_{(r, s)}=o_{(r, s) 2}$ for (odd $r$, odd $s)$ and (even $r$, even $s)$, and $z_{(r, s)}=o_{(r, s) 3}$ for (even $r$, odd $s$ ).

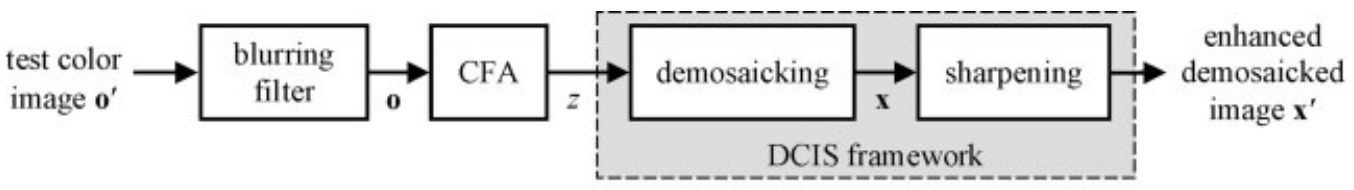

(a)

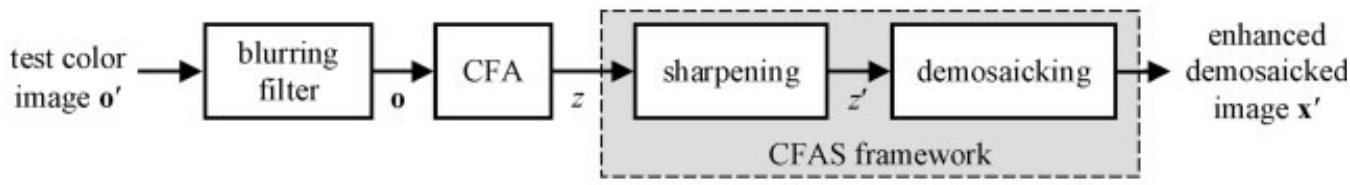

(b)

Figure 6. Evaluation procedure: (a) DCIS framework, (b) CFAS framework. 
Table I. Comparison of the methods using images blurred by a $3 \times 3$ mean filter.

\begin{tabular}{|c|c|c|c|c|c|c|c|c|c|c|c|c|}
\hline \multirow[b]{2}{*}{ Sharpening } & \multicolumn{3}{|c|}{ DCIS } & \multicolumn{3}{|c|}{ CFAS $_{1}$} & \multicolumn{3}{|c|}{$\mathrm{CFAS}_{2}$} & \multicolumn{3}{|c|}{$\mathrm{CFAS}_{3}$} \\
\hline & MAE & MSE & SCD & MAE & MSE & SCD & MAE & MSE & SCD & MAE & MSE & SCD \\
\hline \multicolumn{13}{|c|}{ Demosaicking } \\
\hline$D_{1}$ & 6.770 & 150.3 & 2.493 & 6.207 & 126.8 & 2.110 & 6.399 & 133.3 & 2.366 & 6.203 & 127.8 & 2.112 \\
\hline$D_{2}$ & 6.809 & 154.6 & 2.534 & 6.247 & 128.5 & 2.215 & 6.392 & 133.1 & 2.409 & 6.299 & 132.4 & 2.307 \\
\hline$D_{3}$ & 6.820 & 150.9 & 2.516 & 6.266 & 127.2 & 2.202 & 6.447 & 133.2 & 2.285 & 6.307 & 129.7 & 2.392 \\
\hline$D_{4}$ & 6.865 & 152.5 & 2.556 & 6.285 & 127.8 & 2.211 & 6.465 & 133.7 & 2.372 & 6.331 & 130.8 & 2.316 \\
\hline$D_{5}$ & 6.896 & 154.8 & 2.515 & 6.323 & 129.5 & 2.183 & 6.493 & 135.0 & 2.387 & 6.417 & 134.1 & 2.283 \\
\hline$D_{6}$ & 7.309 & 168.0 & 2.678 & 6.455 & 133.1 & 2.290 & 6.493 & 138.4 & 2.373 & 6.485 & 134.9 & 2.388 \\
\hline$D_{7}$ & 6.691 & 148.7 & 2.397 & 6.158 & 125.7 & 2.115 & 6.355 & 132.0 & 2.371 & 6.209 & 128.8 & 2.236 \\
\hline
\end{tabular}

Results were averaged over the members of the test image database shown in Figure 5.

In the DCIS framework (Fig. 6a), demosaicking followed by color image sharpening were used to transform the $K_{1} \times K_{2} \mathrm{CFA}$ image $z$ to the enhanced demosaicked image $\mathbf{x}^{\prime}$ with the same dimensions. In the proposed CFAS framework (Fig. 6b), image sharpening followed by demosaicking were used to produce the enhanced demosaicked image $\mathbf{x}^{\prime}$ from the CFA data by sharpening the structural content of the CFA image $z$. Various demosaicking solutions, such as $D_{1}$ (Pei and Tam, 2003), $D_{2}$ (Lu and Tang, 2003), $D_{3}$ (Lukac et al., 2004a), $D_{4}$ (Chang and Tang, 2004), $D_{5}$ (Gunturk et al., 2002), $D_{6}$ (Alleysson et al., 2005), and $D_{7}$ (Zhang and Wu, 2005), were employed in both processing pipelines to demosaick the CFA image $z$ (in DCIS) or its enhanced variant $z^{\prime}$ (in CFAS). Since most images are intended for human inspection, the performance of the DCIS and CFAS frameworks were evaluated via visual comparisons of the original color image $\mathbf{o}^{\prime}$ to the enhanced demosaicked image $\mathbf{x}^{\prime}$. Note that this approach is routinely used in various real-life image processing applications. Objective evaluation of the camera image sharpening performance can be found in Tables I and II where the results were evaluated using the well-known mean absolute error (MAE) and mean square error (MSE), and the spatial color difference (SCD) criterion which is described by Wandell (1998).

In the first experiment, the test images were blurred using mean filtering, i.e. the low-pass filter coefficients were set to $q_{(i, j)}=1$, for $(i, j) \in \zeta=\{(r+u, s+v) ;-1 \leq u \leq 1,-1 \leq v \leq 1\}$. In the second experiment, a Gaussian blur was introduced by setting low-pass coefficients via $q_{(r+u, s+v)}=\frac{1}{2 \pi \sigma^{2}} \exp \left(-\frac{u^{2}+v^{2}}{2 \sigma^{2}}\right)$ with $\sigma=1$. It is believed that the low-pass filters used in the experiment match the complex imaging process where images are blurred by the camera optics and the sensor, perhaps also due to the camera motion and insufficient focusing.
Figures 7-9 depict enlarged parts of the original, blurred, and enhanced demosaicked images cropped in areas with interesting structural content. The results allow for the visual comparison of both DCIS and CFAS camera image sharpening frameworks. It can be seen that the CFAS framework produces high-quality images with sharp edges and details when processing images blurred via mean filtering (Fig. 7) or Gaussian filtering (Figs. 8 and 9). Unlike the DCIS framework, the CFAS framework significantly reduces the amount of visual impairments present in the final images. The results produced by DCIS indicate the amplification of zipper effects and other demosaicking artifacts (Figs. 8c and 8e). This is not the case when the proposed CFAS framework is used. As shown in Figs. 8d and 8f, our solution excellently enhances the structural content without noticeable side effects and produces higher visual quality and sharpness than the traditional DCIS framework. Consistently good performance of the CFAS framework is confirmed by the results shown in Figure 9. The best visual quality was obtained when CFAS was followed by the powerful demosaicking solution (e.g. $D_{3}, D_{7}$ ) which uses both the structural and spectral information during processing. Thus, the choice of both the sharpening and demosaicking solutions is essential for obtaining the highest visual quality.

Extensive experimentation summarized in Tables I and II showed that $\mathrm{CFAS}_{1}$ and $\mathrm{CFAS}_{3}$ achieved the best performance among the tested sharpeners. With respect to the demosaicking step, the proposed framework works well with most, if not all, demosaicking solutions. Among the solutions under consideration, the data-adaptive spectral demosaicking schemes $\left(D_{1}, D_{2}, D_{3}, D_{4}\right.$, $\left.D_{7}\right)$ outperformed the frequency-domain solutions $\left(D_{5}, D_{6}\right)$. Since the demosaicking scheme $D_{1}$ is characterized by the best trade-off between performance and complexity (Chang and Tang, 2004), the

Table II. Comparison of the methods using images blurred by a $3 \times 3$ Gaussian filter with $\sigma=1$.

\begin{tabular}{|c|c|c|c|c|c|c|c|c|c|c|c|c|}
\hline \multirow[b]{2}{*}{ Sharpening } & \multicolumn{3}{|c|}{ DCIS } & \multicolumn{3}{|c|}{ CFAS $_{1}$} & \multicolumn{3}{|c|}{$\mathrm{CFAS}_{2}$} & \multicolumn{3}{|c|}{$\mathrm{CFAS}_{3}$} \\
\hline & MAE & MSE & SCD & MAE & MSE & SCD & MAE & MSE & SCD & MAE & MSE & SCD \\
\hline \multicolumn{13}{|c|}{ Demosaicking } \\
\hline$D_{1}$ & 5.523 & 95.6 & 2.058 & 5.366 & 88.7 & 1.898 & 5.726 & 100.1 & 2.229 & 5.086 & 80.5 & 1.794 \\
\hline$D_{2}$ & 5.654 & 103.7 & 2.134 & 5.428 & 90.2 & 2.016 & 5.712 & 99.0 & 2.273 & 5.248 & 86.0 & 2.029 \\
\hline$D_{3}$ & 5.507 & 93.4 & 2.059 & 5.383 & 86.4 & 2.018 & 5.734 & 97.3 & 2.152 & 5.143 & 79.8 & 2.125 \\
\hline$D_{4}$ & 5.537 & 93.9 & 2.105 & 5.392 & 86.7 & 2.004 & 5.744 & 97.5 & 2.225 & 5.158 & 80.2 & 2.018 \\
\hline$D_{5}$ & 5.574 & 95.9 & 2.061 & 5.412 & 87.5 & 1.966 & 5.747 & 97.6 & 2.129 & 5.248 & 83.1 & 1.965 \\
\hline$D_{6}$ & 6.081 & 112.2 & 2.242 & 5.571 & 92.5 & 2.063 & 5.875 & 101.8 & 2.193 & 5.382 & 87.4 & 2.079 \\
\hline$D_{7}$ & 5.345 & 90.4 & 1.932 & 5.244 & 84.1 & 1.921 & 5.631 & 95.7 & 2.093 & 4.992 & 77.3 & 1.930 \\
\hline
\end{tabular}

Results were averaged over the members of the test image database shown in Figure 5. 


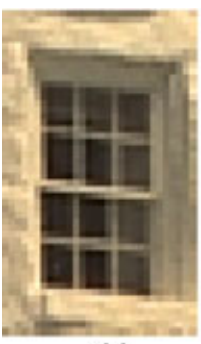

(a)

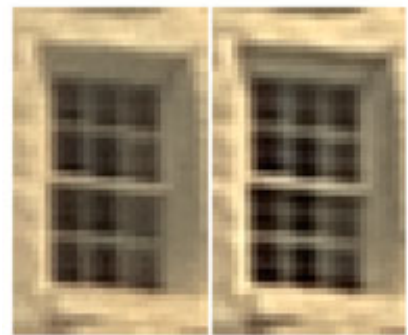

(f)

(b)
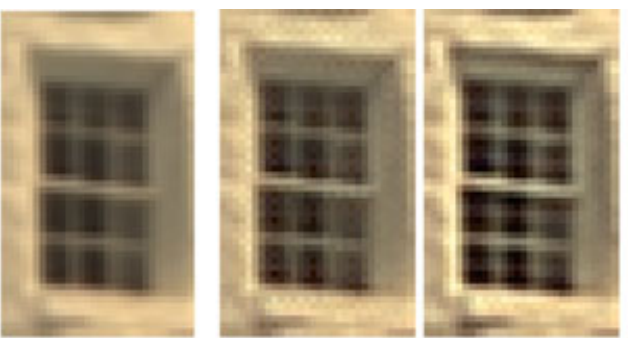

(c)

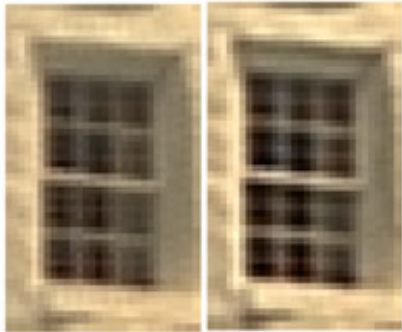

(g)

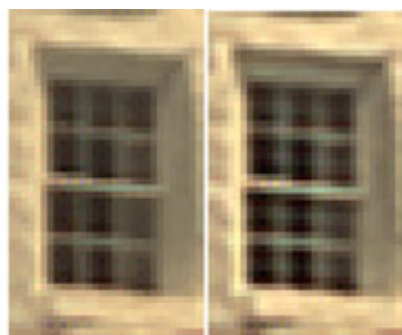

(d)

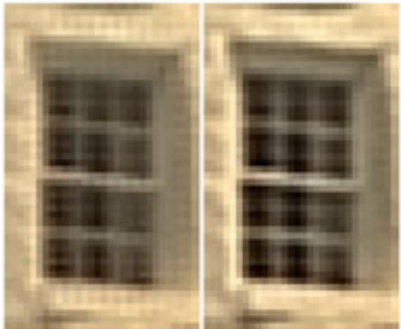

(h)

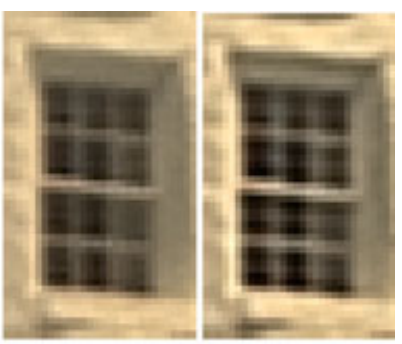

(e)

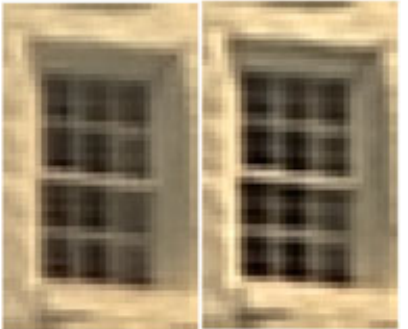

(i)

Figure 7. Cropped $50 \times 30$ parts of the Lighthouse image: (a) original image, (b) image blurred using a $3 \times 3$ mean filter, (c-i) images restored/ enhanced using DCIS (left) and CFAS 1 (right). Employed demosaicking solutions: (c) $D_{1}$, (d) $D_{2}$, (e) $D_{3}$, (f) $D_{4}$, (g) $D_{5}$, (h) $D_{6}$, (i) $D_{7}$. [Color figure can be viewed in the online issue, which is available at www.interscience.wiley.com.]

cascade of $\mathrm{CFAS}_{1}$ or $\mathrm{CFAS}_{3}$ sharpening followed by $D_{1}$ demosaicking constitutes an attractive proposition for cost-effective image-enabled devices such as PDAs.

B. Experimentation Using Original Test Images. To eliminate the influence of the blurring filter employed in the testing pipeline on the produced results, we will now compare the edgeenhancement ability of DCIS and CFAS frameworks through the application of sharpening/demosaicking and demosaicking/sharpening cascades to the non-blurred, 'original' CFA data. Thus, the coefficients of a blurring filter seen in Figure 6 were set to $q_{(i, j)}=1$ for $(i, j)=(r, s)$ and $q_{(i, j)}=0$ for $(i, j) \neq(r, s)$ to perform an identity operation (i.e. no blurring) defined as $o_{(r, s) k}=o_{(r, s) k}^{\prime}$, for $k=1,2,3$. All the remaining steps in the testing pipeline were kept unchanged.

Figures 10 and 11 allow for comparisons of the original, demosaicked, and enhanced demosaicked images. As shown in Figure 10 corresponding to the Water image, the $D_{4}$ demosaicking solution produced high-quality output with well-restored image details and coloration. The only difference between the original image shown in Figure 10a and the demosaicked output shown in Figure 10b can

Figure 8. Cropped $55 \times 60$ parts of the Window image: (a) original image, (b) image blurred using a $3 \times 3$ Gaussian filter with $\sigma=1$, (c) $D_{6}$ followed by DCIS, (d) CFAS ${ }_{1}$ followed by $D_{6}$, (e) $D_{7}$ followed by DCIS, (f) CFAS 1 followed by $D_{7}$. [Color figure can be viewed in the online issue, which is available at www.interscience.wiley.com.]

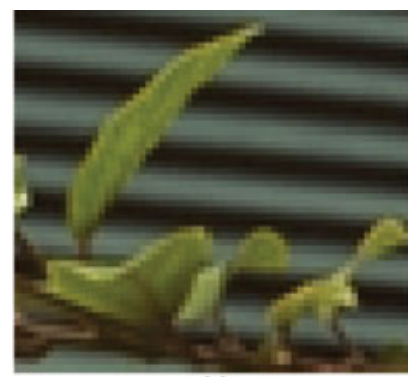

(a)

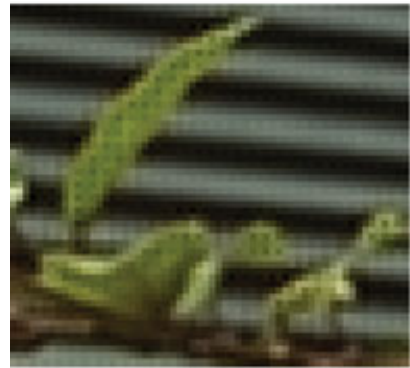

(d)

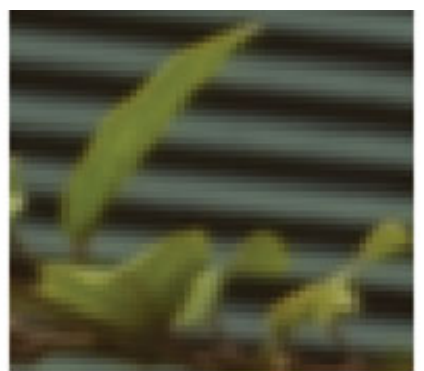

(b)

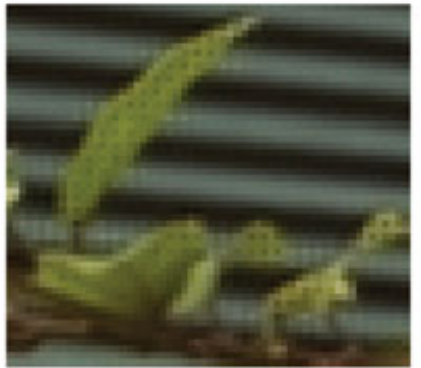

(e)

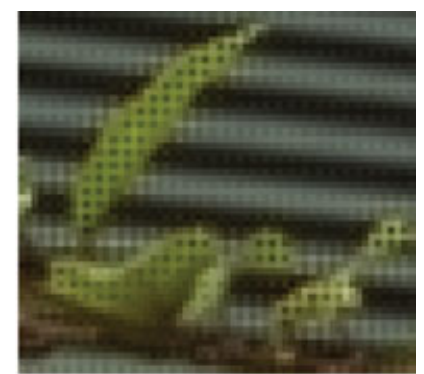

(c)

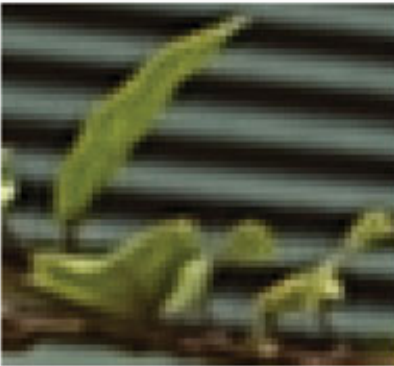

(f) 


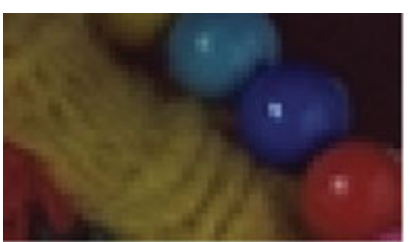

(a)

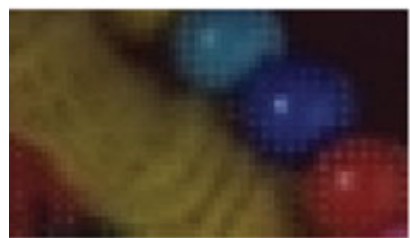

(e)

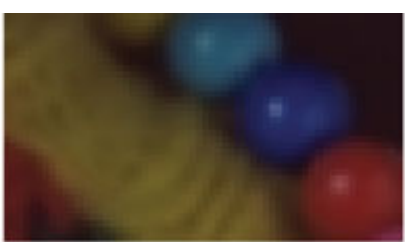

(b)

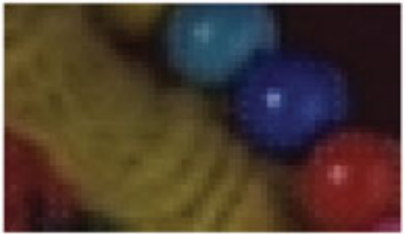

(t)

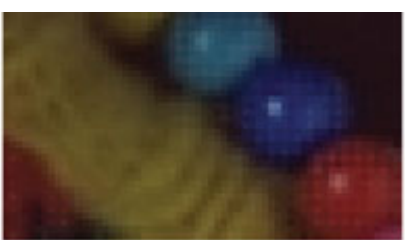

(c)

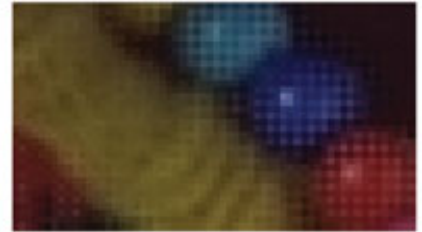

(g)

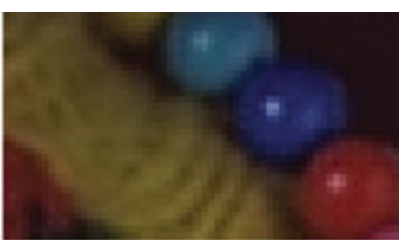

(d)

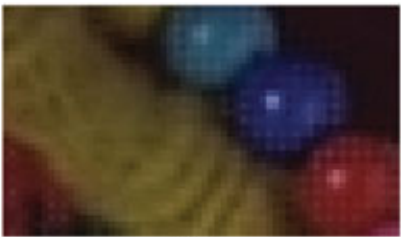

(h)

Figure 9. Cropped $35 \times 60$ parts of the Girl image: (a) original image, (b) image blurred using a $3 \times 3$ Gaussian filter, (c) $D_{1}$ followed by DCIS, (d) CFAS $_{3}$ followed by $D_{1}$, (e) $D_{5}$ followed by DCIS, (f) CFAS 3 followed by $D_{5}$, (g) $D_{6}$ followed by DCIS, (h) CFAS ${ }_{3}$ followed by $D_{6}$. [Color figure can be viewed in the online issue, which is available at www.interscience.wiley.com.]

be seen in the ear area which is affected by zipper effects. Sharpening this demosaicked image using DCIS (Fig. 10c) not only enhances the image sharpness, but also significantly amplifies visual impairments introduced during demosaicking and increases the level of noise present in the boy's face to a disturbing grainy appearance. However, when sharpening is followed by demosaicking, which is the method suggested in the proposed CFAS framework, then the final image (Fig. 10d) has more natural coloration, less noticeable demosaicking artifacts, and does not suffer from noise-like appearance. In addition, inspection of the enhanced demosaicked images shown in Figures 10c and 10d reveal significant improvements in terms of image sharpness in the eyes, teeth, mouth, and eyebrow areas when CFAS is used.

Figure 11 depicts the results corresponding to the Lighthouse image. In this case, the area of interest is a texture from the wall of the lighthouse tower. As shown in Figures $11 \mathrm{a}$ and $11 \mathrm{~b}$, the $D_{7}$ demosaicking solution excellently preserves complex texture edges and fine details. On the other hand, small color shifts can be noticed in the wall texture as well as along a diagonally oriented edge separating the roof and the tower wall. Comparing the images shown in Figures 11c and 11d against the images shown in Figures 11a and $11 \mathrm{~b}$ suggests that both DCIS and CFAS greatly enhanced the edges and fine structural content. However, CFAS clearly outperformed DCIS, as the image shown in Figure 11d is sharper than one seen in Figure 11c and does not suffer from unnatural coloration.

Note that the determination of optimal sharpening and the evaluation of sharpened images are rather difficult and very subjective tasks. For instance, one may consider CFAS enhanced images shown in Figures 10d and 11d over-sharpened compared to DCIS enhanced images shown in Figures 10c and 11c. However, it should not be forgotten that the experimentation presented in this subsection is rather unrealistic due to the omission of the blurring filter from the single-sensor imaging pipeline. Since the blurring filter is a de-facto default element of the camera optical system, the oversharpness currently seen in Figures $10 \mathrm{~d}$ and $11 \mathrm{~d}$ will be reduced to desired sharpness, as it was already proven in the previous subsection through the experimentation using blurred test images. To further verify the issue, camera image sharpeners will be tested next using the real raw single-sensor data.

\section{Experimentation Using Real-Life Raw CFA} Images. Two digital cameras were used: the Canon EOS D-60 camera with a native resolution of $2056 \times 3088$ pixels and the Nikon D70 camera with a native resolution of $2014 \times 3039$ pixels. The input to the simplified single-sensor imaging pipeline, comprised of the demosaicking/DCIS or $\mathrm{CFAS}_{1} /$ demosaicking steps, was obtained by scaling the raw data into 8 bits per CFA entry. In both cases, the $D_{3}$ demosaicking solution was used.

Detailed inspection of the results shown in Figures 12 and 13 indicates that the use of CFAS resulted in higher visual quality compared to DCIS. Namely, Figure $12 \mathrm{~b}$ shows nicely sharpened edges and enhanced details in the horse neck area whereas Figure 12a has these structural contents suppressed. Comparing results shown in Figures 13a and 13b reveals that also in this case CFAS

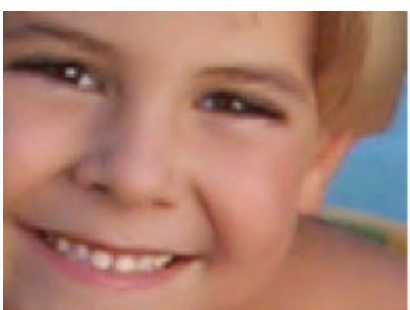

(a)

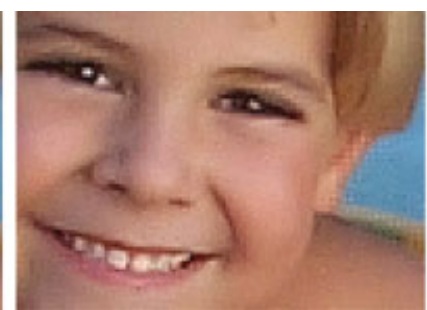

(b)

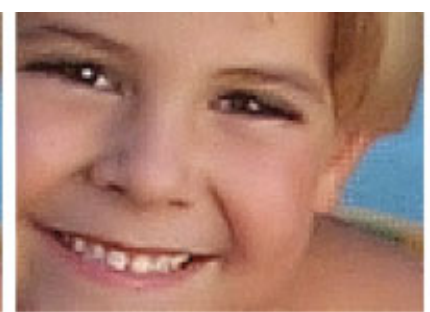

(c)

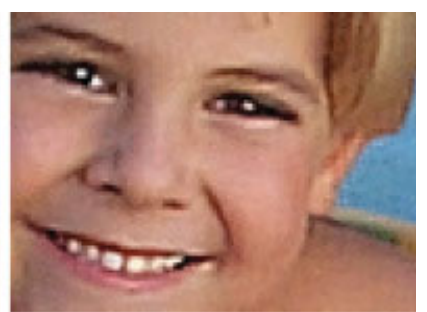

(d)

Figure 10. Cropped $85 \times 115$ parts of the Water image: (a) original image, (b) $D_{4}$, (c) $D_{4}$ followed by DCIS, (d) CFAS 1 followed by $D_{4}$. [Color figure can be viewed in the online issue, which is available at www.interscience.wiley.com.] 


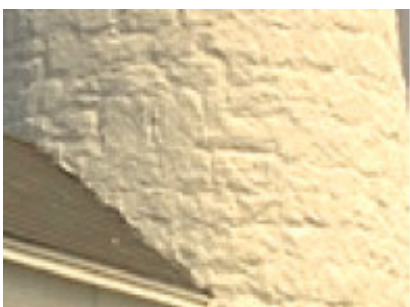

(a)

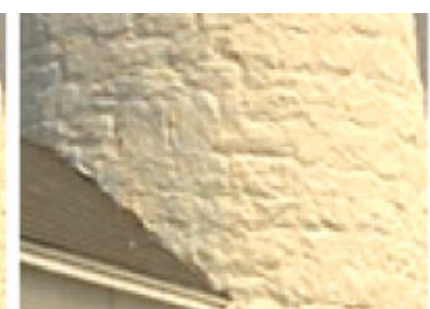

(b)

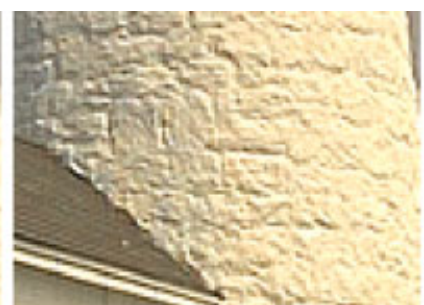

(c)

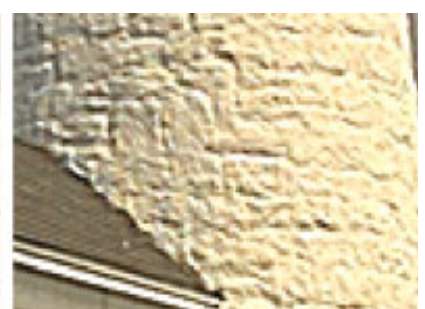

(d)

Figure 11. Cropped $85 \times 115$ parts of the Lighthouse image: (a) original image, (b) $D_{7}$, (c) $D_{7}$ followed by DCIS, (d) CFAS 1 followed by $D_{7}$. [Color figure can be viewed in the online issue, which is available at www.interscience.wiley.com.]

produced the image with enhanced sharpness compared to DCIS. In particular, DCIS degraded the visual quality by increasing the appearance of noise, zipper affects, and other visual impairments such as reduced sharpness, color inconsistency, and visually jagged edges; as can be observed in the bonnet area in Figure 13a. These effects are significantly attenuated or eliminated altogether in Fig-

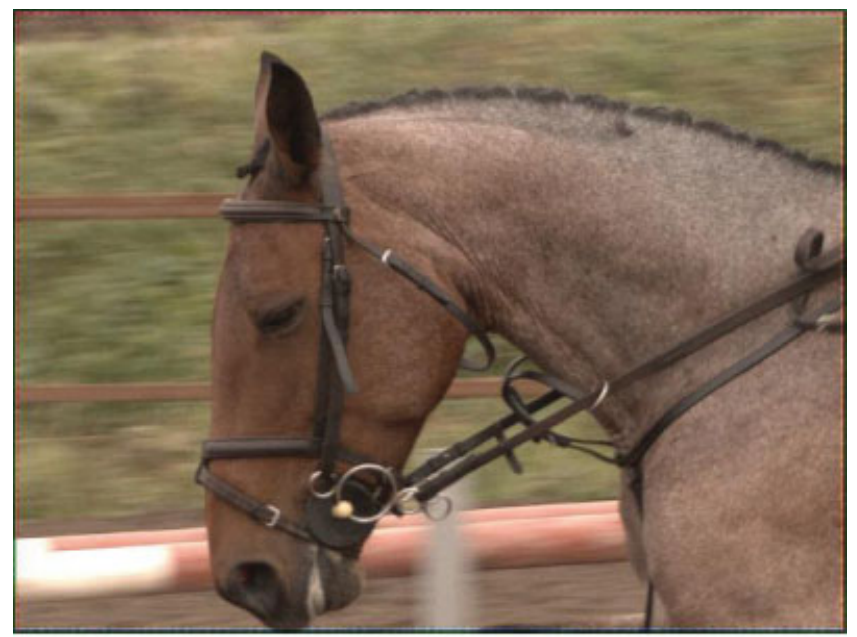

(a)

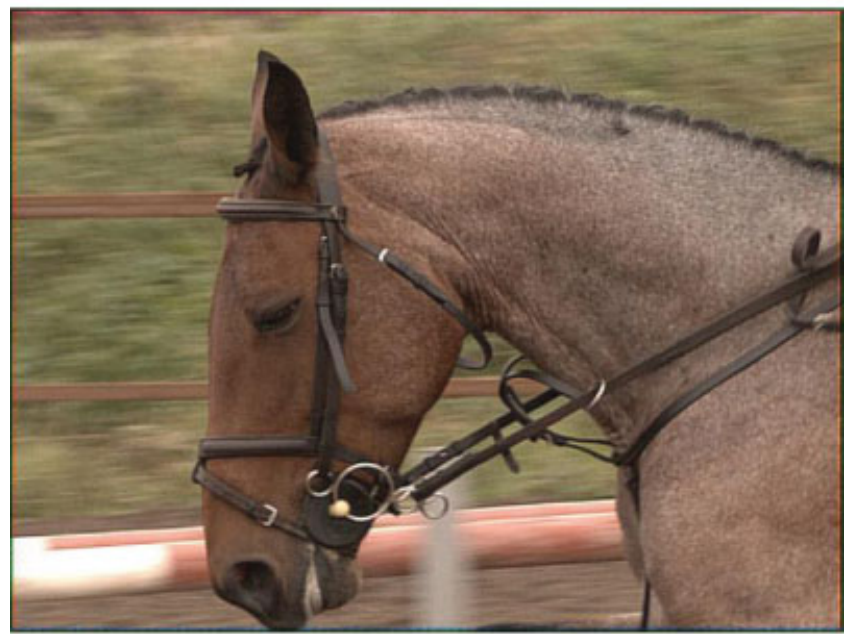

(b)

Figure 12. Cropped $480 \times 640$ patterns of the results obtained using the Horse image: (a) $D_{3}$ followed by DCIS, (b) $\mathrm{CFAS}_{1}$ followed by $D_{3}$. The Horse image is courtesy of Timo Autiokari. ure $13 \mathrm{~b}$, thus demonstrating that CFAS can perform well also in realistic scenarios with raw CFA data.

In summary, the proposed framework: (1) is a viable camera image sharpening solution which enhances the structural content of the acquired CFA image, (2) allows the achievement of the sharp color images directly from subsequent demosaicking, (3)

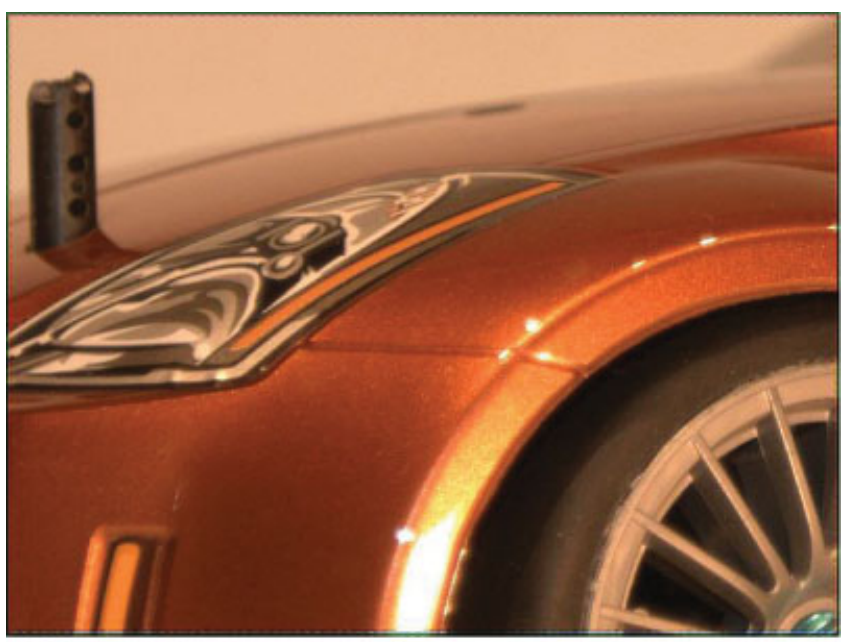

(a)

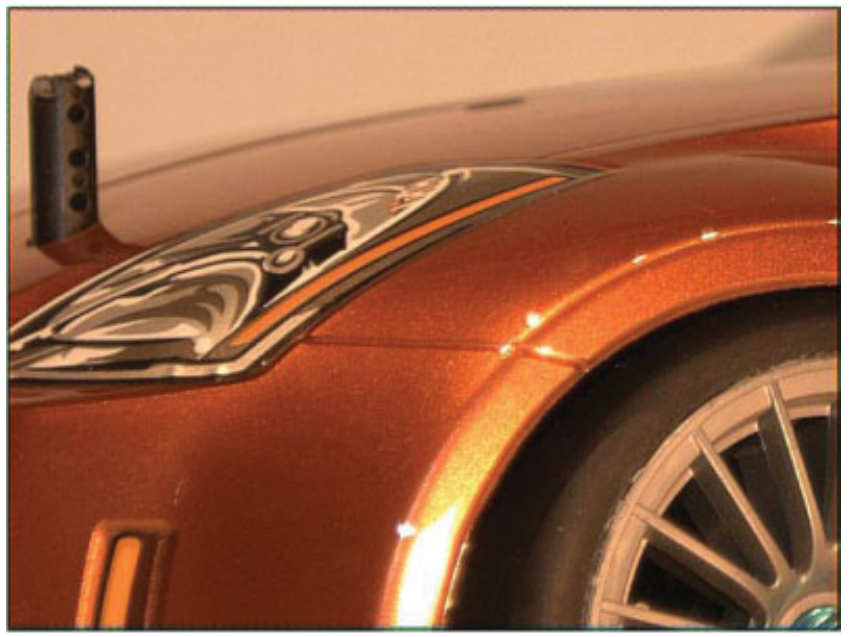

(b)

Figure 13. Cropped $480 \times 640$ patterns of the results obtained using the Car image: (a) $D_{3}$ followed by DCIS, (b) CFAS ${ }_{1}$ followed by $D_{3}$. The Car image is courtesy of Paul Tichonczuk. 
Table III. Camera image sharpening: Average cost per spatial location.

\begin{tabular}{lcccc}
\hline Operation & ADDs & SUBs & DIVs & MULTs \\
\hline DCIS & 3 & 24 & 3 & 3 \\
CFAS $_{1}$ & 1 & 8 & 1 & 1 \\
CFAS $_{2}$ & 1 & 10 & 1 & 1 \\
CFAS $_{3}$ & 1 & 6 & 1 & 1 \\
\hline
\end{tabular}

outperforms the traditional demosaicked image sharpening solution in terms of the image sharpness, and (4) along with demosaicking produce visually pleasing full-color images.

\section{COMPUTATIONAL COMPLEXITY ANALYSIS}

Apart from the actual performance of any algorithm, its computational complexity is a realistic measure of its practicality and usefulness. Therefore, the proposed framework is analyzed here in terms of normalized operations, such as additions (ADDs), subtractions (SUBs), divisions (DIVs), and multiplications (MULTs). Table III summarizes the recorded values needed for the implementation of the various camera image sharpeners. Simple inspection of the recorded values in equivalent normalized operations reveals that the proposed CFAS framework when compared to the traditional DCIS solution achieves a three-fold reduction in computational complexity cost. The implementation efficiency of the proposed framework slightly varies depending on the high-pass filter used to enhance the samples occupying G CFA locations.

The above analysis, as well as execution time measured using a conventional PC equipped with a standard operating system and programming environment indicates that the proposed solution is extremely cost-effective. The execution of the camera image sharpening suite of tools, on an Intel Pentium IV $2.40 \mathrm{GHz}$ CPU, 512 MB RAM box with Windows XP operating system and MS Visual $\mathrm{C}++5.0$ programming environment, took (on average) 0.391 , $0.180,0.191$, and $0.150 \mathrm{~s}$ to enhance a $512 \times 512$ image employing the DCIS, CFAS $1, \mathrm{CFAS}_{2}$, and $\mathrm{CFAS}_{3}$ solutions, respectively. Finally, it should be noted that the objective of this computational complexity analysis is to provide benchmark information regarding implementation issues and not to exhaustively cover all possible implementations. The development of software-optimized realizations of the presented solutions is beyond the scope of this article.

\section{CONCLUSION}

A new single-sensor camera image sharpening framework suitable for enhancing images captured using the Bayer CFA was introduced. The framework operates on the acquired CFA image data in order to improve the sharpness of the demosaicked, full-color image output and boost the computational efficiency of the processing pipeline. Reported result indicated that the proposed here CFA sharpeners outperform traditional solutions, both in terms of performance and efficiency, and produce visually pleasing demosaicked images.

Finally, it should be noted that the scope of this article is limited to the image sharpening component of the single-sensor imaging pipeline. Future research is needed to investigate other single-sensor imaging issues. For example, since the real-life captured images often suffer from noise, image denoising should be used prior to the sharpening step to prevent from amplifying noise and propagating errors down the pipeline to the final output. Also note that the order of demosaicking and sharpening does not guarantee good renditions of color. In the single-sensor pipeline, the coloration of the captured image is usually enhanced using color correction, white balance, and tonescale rendering. Depending on the order of the processing steps, different cameras can produce images with different visual quality.

\section{ACKNOWLEDGMENTS}

The authors would like to acknowledge Timo Autiokari and Paul Tichonczuk for providing the raw CFA single-sensor data used in the experiment.

\section{REFERENCES}

D. Alleysson, S. Süsstrunk, and J. Hèrault, Linear demosaicing inspired by the human visual system, IEEE Trans Image Process 14 (2005), 439-449.

D. Alleysson, S. Süsstrunk, and L. Meylan, HDR CFA image rendering, Proc EURASIP 14th Eur Signal Process Conf, 2006.

B.E. Bayer, Color imaging array, U.S. Patent 3,971,065 (1976).

S. Bouzit and L.W. MacDonald, Colour difference metrics and image sharpness, Proc IS\&T/SID 8th Color Imaging Conf, 2000, pp. 262-267.

L. Chang and Y.P. Tang, Effective use of spatial and spectral correlations for color filter array demosaicking, IEEE Trans Consumer Electron 50 (2004), 355-365.

M. Fischer, J.L. Paredes, and G.R. Arce, Weighted median image sharpeners for the World Wide Web, IEEE Trans Image Process 11 (2002), 717-727.

B. Gunturk, Y. Altunbasak, and R. Mersereau, Color plane interpolation using alternating projections, IEEE Trans Image Process 11 (2002), 997-1013.

B.K. Gunturk, J. Glotzbach, Y. Altunbasak, R.W. Schaffer, and R.M. Murserau, Demosaicking: Color filter array interpolation, IEEE Signal Process Mag 22 (2005), 44-54.

A. Koschan and M. Abidi, Detection and classification of edges in color images, IEEE Signal Process Mag 22 (2005), 64-73.

P. Longére, X. Zhang, P.B. Delahunt, and D.H. Brainard, Perceptual assessment of demosaicing algorithm performance, Proc IEEE 90 (2002), 123-132.

W. Lu and Y.P. Tang, Color filter array demosaicking: New method and performance measures, IEEE Trans Image Process 12 (2003), 1194-1210.

R. Lukac, K.N. Plataniotis, D. Hatzinakos, and M. Aleksic, A novel cost effective demosaicing approach, IEEE Trans Consumer Electron 50 (2004a), 256-261.

R. Lukac, K. Martin, and K.N. Plataniotis, Demosaicked image postprocessing using local color ratios, IEEE Trans Circuit Systems Video Technol 14 (2004b), 914-920.

R. Lukac, B. Smolka, K. Martin, K.N. Plataniotis, and A.N. Venetsanopulos, Vector filtering for color imaging, IEEE Signal Process Mag 22 (2005), 74-86.

R. Lukac and K.N. Plataniotis, Color filter arrays: Design and performance analysis, IEEE Trans Consumer Electron 51 (2005), 1260-1267.

R. Lukac and K.N. Plataniotis, Cost-effective sharpening of single-sensor camera images, IEEE Int Conf Multimedia Expo, 2006, pp. 829-832.

K. Parulski and K.E. Spaulding, "Color image processing for digital cameras', in Digital Color Imaging Handbook, G. Sharma(Editor), CRC Press, Boca Raton, FL, 2002, pp. 728-757.

S.C. Pei and I.K. Tam, Effective color interpolation in CCD color filter arrays using signal correlation, IEEE Trans Circuits Systems Video Technol 13 (2003), 503-513.

A. Polesel, G. Ramponi, and V.J. Mathews, Image enhancement via adaptive unsharp masking, IEEE Trans Image Process 19 (2000), 505-510.

R. Ramanath, W.E. Snyder, Y. Yoo, and M.S. Drew, Color image processing pipeline, IEEE Signal Process Mag 22 (2005), 34-43.

B. Wandell, S-CIELAB: A spatial extension of the CIE L*a*b* DeltaE color difference metric.' Available online at http://white.stanford.edu/ brian/scielab/.

L. Zhang and $\mathrm{X}$. Wu, Color demosaicking via directional linear minimum mean square-error interpolation, IEEE Trans Image Process 14 (2005), 2167-2178. 